\title{
THE CYLINDRICAL MULTI-WIRE SYSTEM TO PROVIDE HOMOGENEOUS ELECTRIC FIELD
}

\author{
Ján Janík — Marian Veselý *
}

\begin{abstract}
This article is a study of electrostatic field homogeneity produced by system of wires cylindrically placed parallel to the axis. The study investigates the homogeneity in a chamber having 20 wires and compares results of different computation methods. The chamber can be a part of a charge particles beam deflecting system as good as it can be an electrostatic part of Wien velocity filter for electron/ion beams. Results of field homogeneity radius calculations are presented.
\end{abstract}

Ke y w ords: electron beam deflection, scanning system for charged particle beam, Wien velocity filter

\section{INTRODUCTION}

Transversal electric field is often used for manipulating with electron/ion beams. As an example we can introduce a cathode ray tube in oscilloscope, electromagnetic spectrometers, charged particle detectors, Wien velocity filter or scanning system of ion implanter, various types of beam deflectors, electron beam lithographs, etc. Here we need a very precise correlation between the beam displacement and the electric field. Usually the beam deflection occurs between two parallel plates. Unfortunately, the field between real plates is no ideal due to existing fringing fields. To improve the electric field homogeneity, some additional plates are placed at sides [1] in the neighbourhood of main plates. But also other electrode systems do exist which can produce a homogeneous electric field. One of such systems is a cylindrical multi-wire chamber (CMWC). The idea to improve the field by auxiliary electrodes is rather old. In process of developing and improving cathode ray tubes for TV a various such arrangements were designed [2]. The mentioned CMWC uses for it the wires. In our arrangement it consists of parallel wires cylindrically placed around axis [3]. Potentials of the wires have cosinusoidal/sinusoidal distribution. This system has many good properties. From a construction point of view the electric field in CMWC is not connected with fixed orientation as it is in the case of capacitor parallel plates. Therefore a field strength vector can have an arbitrary direction depending only on wire potential. It means that such an electrode system can deflect beam in various directions. Then also creation of a rotary electric field is possible. The next good property of a CMWC is the independence of electric field vector orientation on wire mechanical arrangement. The plane capacitor has the vector oriented always perpendicular to plate surface. In conventional $X Y$ sweeping system one needs two pairs of planar electrodes. But that $X Y$ sweeping and even spiral sweeping is possible in one CMWC
[4]. Also magnetostatic version of spiral sweeping is possible [5]. Next good property - the field in the CMWC is only a little dependent on presence of protuberant metal parts. This property is very useful in Wien velocity filter where the magnet metal pole tips can affect the electric field homogeneity. In literature a multiwire chamber which is used as a particle detector in high energy physics [6] is well known. Such chambers contain gases. Also some technological processes used at our Institute need a large scale homogeneous electric field in cylindrical quartz pipe and in presence of a gas. We mention here only oriented growth of carbon nanotubes [7] for cold cathodes [8]. One of aims of this article will be to estimate the minimum number of wires giving a sufficient field homogeneity.

\section{CYLINDRICAL MULTI-WIRE ELECTROSTATIC CHAMBER}

A creation of desired in our case a homogeneous electric field - is based on idea illustrated in Fig. 1. If in a homogeneous field some virtual closed area $\Omega$ is inserted with defined potential distribution $\varphi(\Omega)$, then inside it there will be also a homogeneous field. The area $\Omega$ can be realized by inserting mutual insulated metal parts. The metal parts cannot fully cover the area (metal parts have different potentials). It is a reason, why the field inside an area is disturbed. The disturbance will be smaller when we increase the number of auxiliary metal parts [9]. The ideal (eg CMWC having many thin wires) multiwire chamber fully fulfils this idea. The real CMWC has restricted number of wires in a cylinder of radius $R_{r}$. The radii of wires are smaller than the cylinder radius (Fig.2). The wire potentials are

$$
\varphi(k)=\varphi_{0} \cos \vartheta_{k}
$$

where $\vartheta_{k}$ is an angle between $x$ axis and wire radiusvector.

\footnotetext{
* Institute of Electronics and Photonics, Faculty of Electrical Engineering and Information Technology, Slovak University of Technology, Ilkovičova 3, 81219 Bratislava, Slovakia, jan.janik@stuba.sk
} 

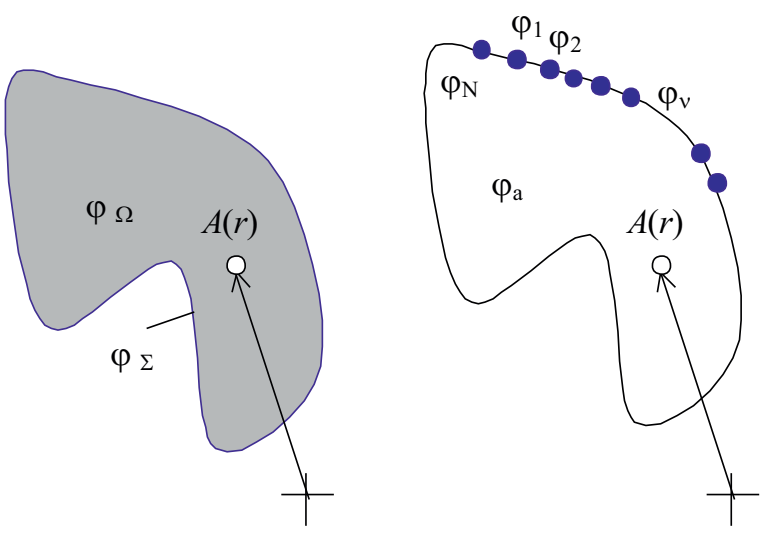

Fig. 1. To idea of a homogeneous field creation

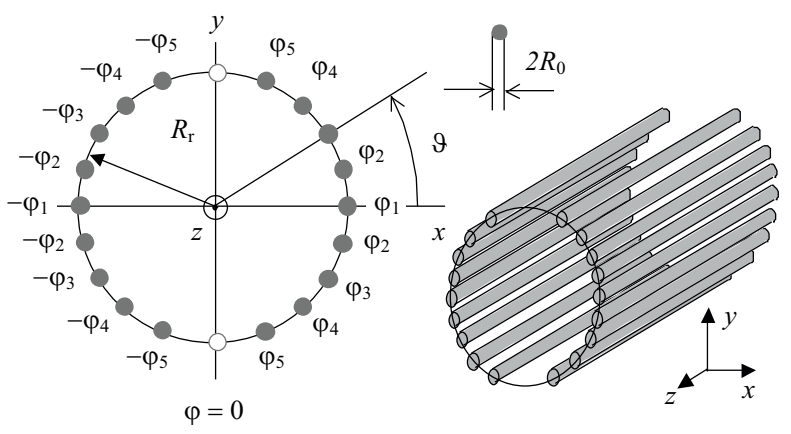

Fig. 2. Practical CMWC configuration

\subsection{Homogeneity criteria}

The homogeneity criteria could be defined to fulfil the desired task. We will define an area of homogeneity where the electric field $E_{x}$ has a difference of less than $1 \%$ of the field $E_{x 0}$ in the centre of CMWC. Mentioned definition of the homogeneity is better visualized in spite of very often used values of harmonic analysis of multiple amplitudes. The multiple amplitudes (usually Fourier series) are also suitable for characterisation of field homogeneity. We can introduce a radius of homogeneity $(\mathrm{HR})$ as a radius of a circle inscribed into the area of homogeneity.

\subsection{Field calculation}

Our first calculations were made in time, when a commercial program codes were not available. The calculations were based on a model of a few wires having linear charge density $\tau$. Then we can calculate the potential at arbitrary point which has a distance $r$ from the wire. As an boundary condition can be assumed the potential on wire surface. Generally it will give us a system of linear equations (2), from which the $\tau_{i}$ can be found.

$$
\varphi_{i}=-\frac{\tau_{i}}{2 \pi \varepsilon_{0}} \ln \left(r_{i}\right)+C\left(r_{i}\right)
$$

Here $i$ is the wire index and $\varphi_{i}$ is its potential. Constant $C(r)$ is chosen a manner to have the potential value equal to $\varphi_{i}$. Then potential at point $A\left(x_{0}, y_{0}\right)$, Fig. 3 , is

$$
\varphi(A)=\frac{\tau}{2 \pi \varepsilon_{0}} \ln \left(\frac{r_{0}}{R}\right)
$$

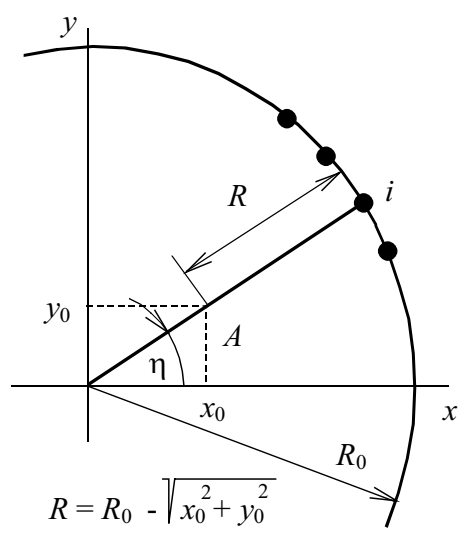

Fig. 3. Contribution to the potential in point $A$ from $i$-th linear charge

Point $A$ has coordinates $x_{0}=R_{0} \cos \eta$ and $y_{0}=$ $R_{0} \sin \eta$

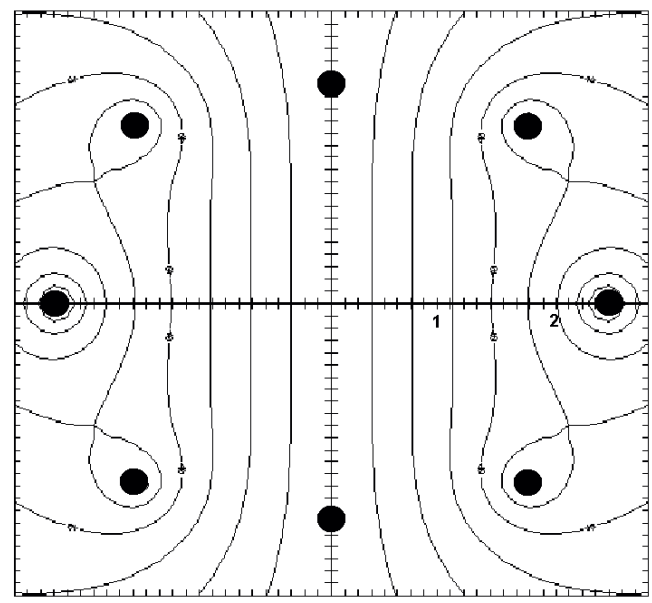

Fig. 4. Map of potential field lines of 8 CMWC

We can assume that linear charge density of a filament is placed in the centre of a real wire and the wire surface to be an equipotential surface. It is true only for chamber diameter much-much larger than the wire diameter. We assume that an error from this approximation is of the order of their ratio. The potential is an additive quantity. Then each wire will contribute in desired point $A$ and the potential at point $A(x, y)$ is given by a sum of all terms of $2 \mathrm{~N}$ wires having a potential $\varphi(k)$. Then the potential at each point inside the CMWC can be very easily calculated by contribution of all these wires

$$
\begin{array}{r}
\varphi_{A}=\frac{\tau_{1}}{2 \pi \varepsilon} \ln \left(\frac{R_{0}}{R_{1}}\right) \frac{\tau_{2}}{2 \pi \varepsilon_{0}} \ln \left(\frac{R_{0}}{R_{2}}\right)+\ldots \\
+\frac{\tau_{N}}{2 \pi \varepsilon_{0}} \ln \left(\frac{R_{0}}{R_{2 N}}\right) .
\end{array}
$$




\subsection{Field homogenity and the number of wires}

The simplest type of CMWC consists of two wires having a potential $\pm \varphi_{0}$. It is possible to express the electric field by a well known analytical formula. Very similar field will be in 4 wire-chamber, because top and bottom electrode potentials are equal to zero. If we neglect the influence of these wires, the field in the vicinity of center in $x$ direction will be very similarly like the field of 2wire chamber. Because such a chamber has no practical application, we shall not study the field in this chamber in more details.

\subsubsection{The eight wire chamber}

All calculations were now made by commercial program OPERA/TOSCA [10]. The CMWC having 8 wires has a cross section shown in Fig. 5. Field on patch between the electrodes is also shown here. The maximum potential is $\varphi_{0}=100 \mathrm{~V}$.
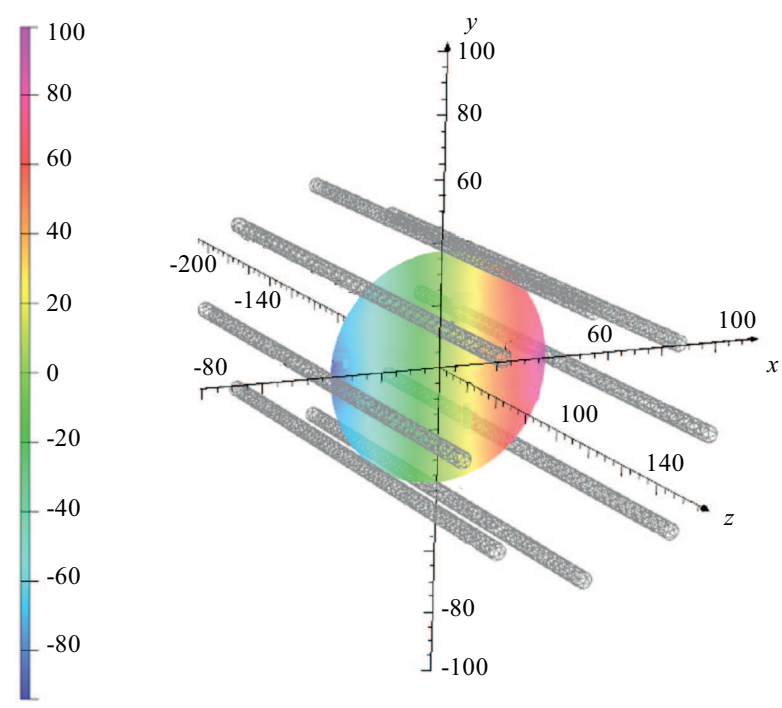

Fig. 5. Field of 8 wire CMWC

An electric field in this chamber has a far more better field homogeneity as it was in the previous one. But the homogeneity radius is evidently small. As a good compromise between minimum of wires and radius of homogeneity seems to be a twenty wire chamber.

\subsubsection{The twenty wire chamber}

In Fig. 6 potential field lines created by 20 charged wires are shown. Because the wires should be inserted into a metallic pipe, we insert this demand into the input deck of OPERA pre-processor. As it can be seen, the field has less unhomogeneity than in previous case. The calculation was made for $R_{0}=4 \mathrm{~cm}, r_{0}=2.5 \mathrm{~mm}$, $R_{\text {pipe }}=5 \mathrm{~cm}$ and $\varphi_{0}=100 \mathrm{~V}$.

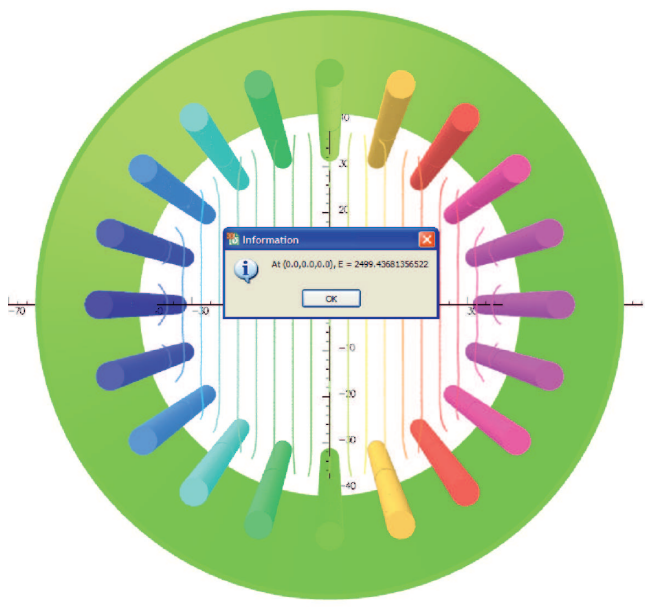

Fig. 6. View of 20 wire CMWC modelled by OPERA

For this 20 wire CMWC we will look in more details for this field strength. The electrostatic field was calculated also by commercial program IGUN [11]. The area of homogeneity is shown in Fig. 7 .

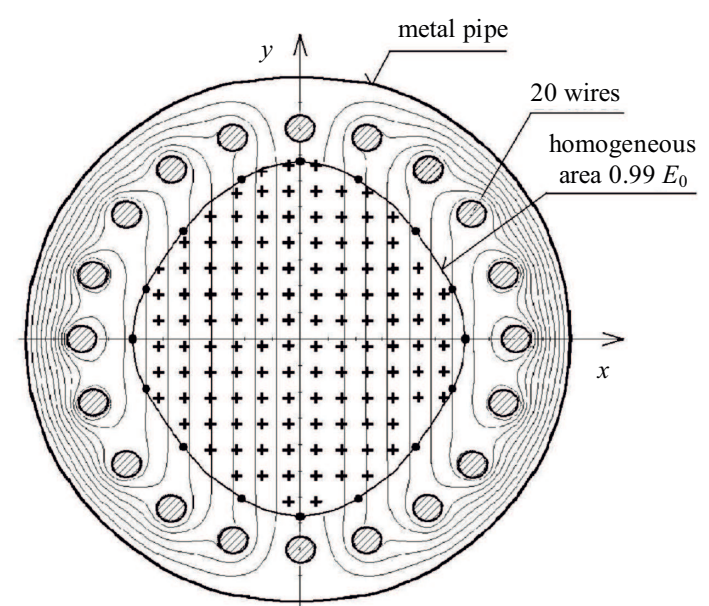

Fig. 7. View of 20 wire CMWC modelled by OPERA

We will compare the field strength calculated by various computer programs (own program, IGUN, OPERA, and equations [2]). The graph is shown in Fig. 8.

For various wire numbers the area of homogeneity was calculated. A dependence of radius of homogeneity $\mathrm{RH}$ on number of wires is shown in Fig. 9. Here is RH given as ratio of $\mathrm{RH}$ and radius of circle where wires are placed

\section{CONCLUSION}

The calculated results of field strength in axis vicinity made by OPERA and IGUN are almost identical. Calculations by own code are different by about $3 \%$ and lie between values of ideal planar plates and values given by commercial programs. Previous calculation published by Salinger [2] lies about $30 \%$ higher. We are convinced, our calculation is more precise. Therefore it is possible to use for calculation any program. CMWCHs are a good alternative for beam displacement/scanning system to plan 


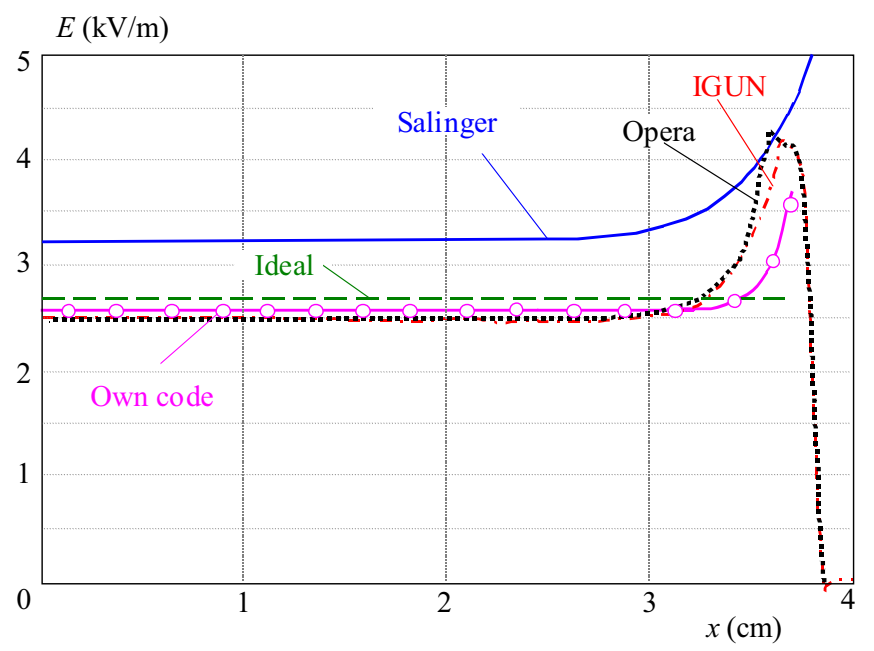

Fig. 8. Comparison of electric field calculations

parallel plate system usually used for beam deflection. The number of 20 wires seems to be a good compromise in regard to a radius of homogeneity. As we can see from Fig. 7 and Fig. 9, our calculations and OPERA calculation are in good agreement. We should mention the fact, that all calculations presented in this article were made without taking into account the space charge effects induced by ion/electron beam. A more detailed study of $X Y$ sweeping system for electron/ion beam, including beam space charge effects as well as parallel displacement systems, is being carried on and is already well elaborated.

\section{Acknowledgemet}

This work was partially supported by Project - Centre of Excellence CENAMOST (Agency for supporting of science an research, Contract No. VVCE-0049-07), by VEGA project $1 / 1102 / 11$ and $1 / 0807 / 08$. We are grateful to S. Bederka for giving us a possibility to elaborate this problem, to provide us with preliminary results. We thank him for consultations and his permanent interest in this problem. Our thanks belong to P. Gonda and J. Franek for valuable discussions.

\section{REFERENCES}

[1] DEARNAley, G.-FreEman, J. H.-NELSON, R. S.-STEPHEN, J. : Ion Implantation, Nord-Holland Publishing Comp., Amsterdam, London 1973.

[2] SAlingeR, H. W.: The electrostatic dissector, Proc. Nat. Elect. Conf. No. II, 82-88, October 3-5, 1946 (USA).

[3] BEDERKA, Š. : An electrostatic (x,y) deflection system for high current beams, ATOMKI Kzlemnyek No. 18 (1976), 431-437.

[4] BEDERKA, S. : Elektrostatick vychylovacia komora, Cs. patent AO 177549, (Electrostatic deflecting chamber, Cs. Patent) PRAHA (1979)..

[5] AKHMetOV, T. D.: Rev. Sci. Istrum., 7703 C106 (2006).

[6] VINDUŠKA, P.—JANÍK. J.-BÚC, D. : Investigation of Condition for Preparation of Oriented nanotubes at Department of Microelectronics in a Modified Plasma Enhancement Hot Filament Chemical Vapour Deposition Reactor, Physica E 40 (2008), 2247-2251.

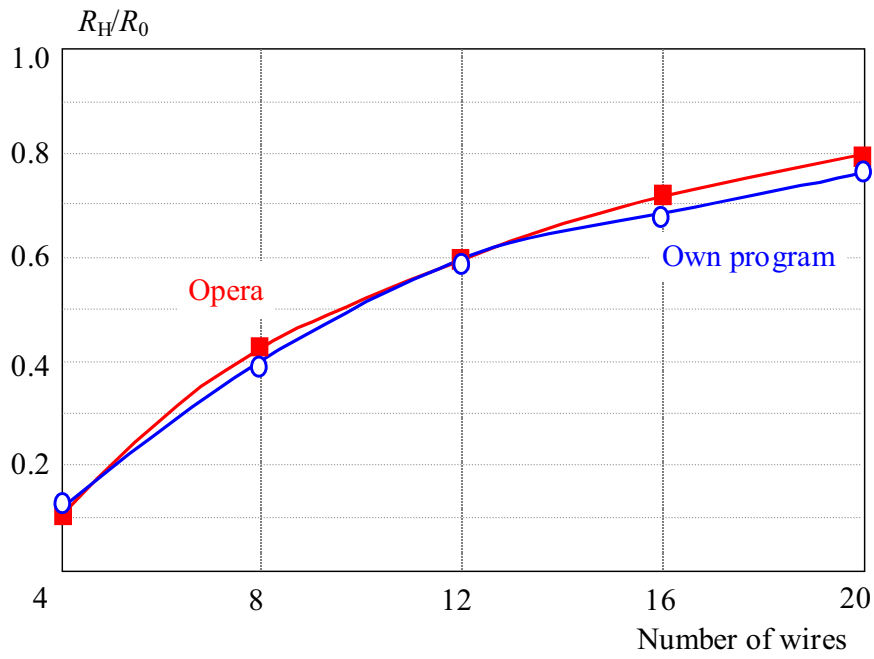

Fig. 9. Radius of homogeneity

[7] JANÍK. J.-BALON, F.—VINDUŠKA, P.—DANIŠ, T. : Field and Electron Trajectory Modelling in Vicinity of an Emitting Tip, Journal of Vacuum Science and Technology. B. 24 No. 4 (2006), 1951-1955.

[8] FRANEK, J.-JANČÁRIK, V.-KOLLÁR, M. : Remarks on homogeneous electrostatic field (Unpublished presentation). Private communication..

[9] FRANEK, J.-KOLLÁR, M. : Solution of a Case of Static Field with Mixed Boundary Conditions, Elektro 2010 Proceedings of the 8-th International Conference, Žilina, Slovakia, 24-25 May 2010, CD-Rom..

[10] OPERA, http://www.cobham.com/about-cobham/avionicsand-surveillance/about-us/technical-services/kidlington /products/opera-3d - electromagnetic-design-in-threedimensions.aspx.

[11] BECKER, R.-HERRMANNSFELDT, B. W. : IGUN, A computer code for the simulation of positive ion extraction including magnetic field. Rev. Sci. Instrum. 63940 April 1992.

Received 2 May 2012

Ján Janík (Assoc Prof, Ing, CSc) was born in Handlová in 1946. He received his Ing (MSc) degree in solid state physics, in 1970 and the CSc (PhD) in applied physics, in 1981, both form the Slovak University of Technology, Bratislava. Since 1992 he has been Associate Professor in Electronics (thesis: The Methodics of Ionooptical Elements and Systems Computation) at the Departmnet of Microelectronics of Slovak University of Technology. He tought different subjects as vacuum physics and technology, optoelectronics, microwave technology, electrical instrumentation, electron and ion technology, electronic and microelectronic elements, and microelectronic materials technology. In research he is oriented to ion beam optics and plasma technology.

Marian Veselý (Assoc. Prof., Dr.), graduated from STU, Faculty of Electical Engineering (FEE) in 1967. He has completed also studies at University of Illinois with a MSc degree. He got his PhD at STU in Electronics and Vacuum Physics in 1972. Since the graduation he has hold research and later teaching positions at the Institute of electronics and Photonics, Faculty of Electrical Engineering and Information technology, Slovak University of Technology in Bratislava. His area of research interest is diamond and DLC technology and related topics. 